\title{
A NOTE ON A PAIR OF DERIVATIONS OF SEMIPRIME RINGS
}

\author{
MUHAMMAD ANWAR CHAUDHRY and A. B. THAHEEM
}

Received 17 February 2003

\begin{abstract}
We study certain properties of derivations on semiprime rings. The main purpose is to prove the following result: let $R$ be a semiprime ring with center $Z(R)$, and let $f, g$ be derivations of $R$ such that $f(x) x+x g(x) \in Z(R)$ for all $x \in R$, then $f$ and $g$ are central. As an application, we show that noncommutative semisimple Banach algebras do not admit nonzero linear derivations satisfying the above central property. We also show that every skew-centralizing derivation $f$ of a semiprime ring $R$ is skew-commuting.
\end{abstract}

2000 Mathematics Subject Classification: 47A50, 47B50.

1. Introduction and preliminaries. Throughout, $R$ denotes a ring with center $Z(R)$. We write $[x, y]$ for $x y-y x$. We will frequently use the identities $[x y, z]=x[y, z]+$ $[x, z] y$ and $[x, y z]=y[x, z]+[x, y] z$ for all $x, y, z \in R$. We recall that $R$ is semiprime if $a R a=(0)$ implies $a=0$ and it is prime if $a R b=(0)$ implies $a=0$ or $b=0$. A prime ring is semiprime but the converse is not true in general. An additive mapping $d: R \rightarrow R$ is called a derivation if $d(x y)=d(x) y+x d(y)$ for all $x, y \in R$. A mapping $f: R \rightarrow R$ is called centralizing if $[f(x), x] \in Z(R)$ for all $x \in R$; in particular, if $[f(x), x]=0$ for all $x \in R$, then it is called commuting. A mapping $f: R \rightarrow R$ is called central if $f(x) \in Z(R)$ for all $x \in R$. Every central mapping is obviously commuting but not conversely, in general. A lot of work has been done on centralizing mappings (see, e.g., $[3,4,5]$ and the references therein). A mapping $f: R \rightarrow R$ is called skew-centralizing if $f(x) x+x f(x) \in Z(R)$ for all $x \in R$; in particular, if $f(x) x+x f(x)=0$ for all $x \in R$, then it is called skew-commuting. We denote the radical of a Banach algebra $A$ by $\operatorname{rad}(A)$.

We now recall some facts concerning semiprime rings and their extended centroids. For any semiprime ring $R$, one can construct the ring of quotients $Q$ of $R$ [1]. As $R$ can be embedded isomorphically in $Q$, we consider $R$ as a subring of $Q$. If the element $q \in Q$ commutes with every element in $R$, then $q$ belongs to $C$, the center of $Q$. $C$ contains the centroid of $R$ and is called the extended centroid of $R$. In general, $C$ is a von Neumann regular ring, and it is a field if and only if $R$ is a prime [1, Theorem 5]. For more information on extended centroid of $R$, we refer to [2].

Brešar [6, Theorem 2] has proved that if $R$ is a prime ring of characteristic not 2 and $f: R \rightarrow R$ is an additive skew-commuting mapping (i.e., $f$ satisfies $f(x) x+x f(x)=0$ for all $x \in R$ ), then $f=0$.

Moreover, Brešar [5, Theorem 4.1] has considered a pair of derivations on a prime ring and has proved the following. Let $R$ be a prime ring and $U$ a nonzero left ideal of $R$. Suppose that the derivations $d$ and $g$ of $R$ are such that $d(u) u-u g(u) \in Z(R)$ for all $u \in U$. If $d \neq 0$, then $R$ is commutative. 
A mapping $h: R \rightarrow R$ defined by $h(x)=a x+x b(x \in R)$ for some $a, b \in R$ is called a generalized inner derivation [8]. Generalized inner derivations are called elementary operators and have been extensively studied in operator algebras. We note that the condition that $h$ is centralizing on $R$ can be written in the form $[a, x] x+x[b, x] \in Z(R)$ for all $x \in R$. Thus, introducing inner derivations $f$ and $g$ by $f(x)=[a, x]$ and $g(x)=$ $[b, x]$, we obtain the condition as in [5, Theorem 4.1], that is, $f(x) x+x g(x) \in Z(R)$ for all $x \in R$.

Recently, Thaheem [9] has proved the following result.

THEOREM 1.1. If $f, g$ is a pair of derivations on a semiprime ring $R$ satisfying $f(x) x+$ $x g(x)=0$ for all $x \in R$, then $f(x), g(x) \in Z(R)$ and $f(u)[x, y]=g(u)[x, y]=0$ for all $u, x, y \in R$.

Inspired by the works of Brešar [5, 6] and Thaheem [9] and the above remarks regarding generalized inner derivations, we consider a general situation regarding a pair of derivations of a semiprime ring and prove the following. Let $f, g$ be a pair of derivations of a semiprime ring $R$ satisfying $f(x) x+x g(x) \in Z(R)$ for all $x \in R$, then $f$ and $g$ are central (Theorem 2.2). We also show that every skew-centralizing derivation $f$ of a semiprime ring $R$ is skew-commuting (Corollary 2.3).

We will need the following result of Brešar [7, Theorem 3.1] in the sequel.

THEOREM 1.2. Let $S$ be a set and $R$ a semiprime ring. If functions $f$ and $g$ of $S$ into $R$ satisfy $f(s) \times g(t)=g(s) \times f(t)$ for all $s, t \in S, x \in R$, then there exist idempotents $\epsilon_{1}, \epsilon_{2}, \epsilon_{3} \in C$ and an invertible element $\lambda \in C$ such that $\epsilon_{i} \epsilon_{j}=0$, for $i \neq j, \epsilon_{1}+\epsilon_{2}+\epsilon_{3}=1$, and $\epsilon_{1} f(s)=\lambda \epsilon_{1} g(s), \epsilon_{2} g(s)=0, \epsilon_{3} f(s)=0$ hold for all $s \in S$.

2. The results. We now prove our results.

LEMMA 2.1. Let $f, g$ be a pair of derivations of a semiprime ring $R$ satisfying $f(x) x+$ $x g(x) \in Z(R)$, then $c f$ and $c g$ are central for all $c \in Z(R)$.

Proof. If $c=0$, then obviously $c f$ and $c g$ are central. Let $c$ be a nonzero element of $Z(R)$. Linearizing $f(x) x+x g(x) \in Z(R)$, we get

$$
f(x) y+f(y) x+x g(y)+y g(x) \in Z(R) \quad \forall x, y \in R .
$$

Taking $y=c$ in (2.1), we get

$$
f(x) c+f(c) x+x g(c)+c g(x) \in Z(R) \quad \forall x \in R .
$$

Replacing $y=c^{2}$ in (2.1), we obtain

$$
f(x) c^{2}+2 c f(c) x+x(2 c g(c))+c^{2} g(x) \in Z(R),
$$

that is,

$$
c[f(x) c+c g(x)+f(c) x+x g(c)]+c[f(c) x+x g(c)] \in Z(R) .
$$


Noting that the first summand is contained in $Z(R)$ by (2.2), from (2.4), we obtain

$$
c[f(c) x+x g(c)] \in Z(R) \quad \forall x \in R .
$$

Thus

$$
[c(f(c) x+x g(c)), y]=0 \quad \forall x, y \in R .
$$

This implies

$$
c[f(c) x+x g(c), y]=0 \quad \forall x, y \in R .
$$

Further, (2.2) implies

$$
[f(x) c+c g(x), y]=-[f(c) x+x g(c), y] \quad \forall x, y \in R
$$

From (2.7) and (2.8), we obtain $c[f(x) c+c g(x), y]=0$, which implies

$$
c^{2}[f(x)+g(x), y]=0 \quad \forall x, y \in R
$$

Replacing $y$ by $z y$ in (2.9), we get $c^{2} z[f(x)+g(x), y]=0$, which implies

$$
c z c[f(x)+g(x), y]=0 \quad \forall x, y, z \in R .
$$

Replacing $z$ by $[f(x)+g(x), y] z$ in (2.10), we get $c[f(x)+g(x), y] z c[f(x)+g(x), y]=$ 0 , which, by semiprimeness of $R$, implies $c[f(x)+g(x), y]=0$; that is, $[c(f(x)+$ $g(x)), y]=0$ for all $x, y \in R$. Thus,

$$
c[f(x)+g(x)] \in Z(R) \quad \forall x \in R .
$$

Since $c \in Z(R)$ and $f, g$ are derivations, therefore $c f, c g$, and $c(f+g)$ are derivations of $R$. Further, (2.11) implies that $c(f+g)$ is central and hence, by [3, Lemma 4], a commuting derivation. Thus, by Thaheem and Samman [10, Proposition 2.3], we get $(c(f+g))(u)[x, y]=0$ for all $u, x, y \in R$. That is,

$$
c(f(u)+g(u))[x, y]=0 \quad \forall u, x, y \in R .
$$

Using (2.12) and the fact that $c f(u)+c g(u) \in Z(R)$, we get $[(c f(u)+c g(u)) u, y]=$ $(c f(u)+c g(u))[u, y]+[c f(u)+c g(u), y] u=0$; that is,

$$
[c f(u) u+c g(u) u, y]=0 \quad \forall u, y \in R .
$$


Since $c \in Z(R)$ and $f(u) u+u g(u) \in Z(R)$, therefore $c f(u) u+c u g(u) \in Z(R)$. Thus

$$
[c f(u) u+c u g(u), y]=0 \quad \forall u, y \in R .
$$

Subtracting (2.14) from (2.13), we get $[c g(u) u-c u g(u), y]=0$; that is, $[c(g(u) u-$ $u g(u)), y]=[c[g(u), u], y]=[[c g(u), u], y]=0$ for all $u, y \in R$, which implies $[c g(u), u] \in Z(R)$. Thus, $c g$ is a centralizing derivation. By [3, Lemma 4], we get that $c g$ is a commuting derivation. By Thaheem and Samman [10, Proposition 2.3], we get $c g(u) \in Z(R)$. Thus $c g$ is central.

Since $c f(u)+c g(u) \in Z(R)$ and $c g(u) \in Z(R)$, therefore $c f(u) \in Z(R)$. So $c f$ is central.

THEOREM 2.2. Let $R$ be a semiprime ring and $f, g$ a pair of derivations of $R$ such that $f(x) x+x g(x) \in Z(R)$ for all $x \in R$. Then $f$ and $g$ are central.

Proof. Let $x_{0} \in R$ and $c=f\left(x_{0}\right) x_{0}+x_{0} g\left(x_{0}\right)$. Then, by hypothesis, $c \in Z(R)$. By Lemma 2.1, $c f$ and $c g$ are central. Thus $[c f(x), y]=0$ for all $x, y \in R$. That is, $c f(x) y-y c f(x)=0$, which implies

$$
f(x) y c=c y f(x) \quad \forall x, y \in R .
$$

Taking $S=R, g(x)=c$ and applying Theorem 1.2 to (2.15), we get that there exist idempotents $\epsilon_{1}, \epsilon_{2}, \epsilon_{3} \in C$ and an invertible element $\lambda \in C$ such that $\epsilon_{i} \epsilon_{j}=0$ for $i \neq j$, $\epsilon_{1}+\epsilon_{2}+\epsilon_{3}=1$, and

$$
\epsilon_{1} f(x)=\lambda \epsilon_{1} c, \quad \epsilon_{2} c=0, \quad \epsilon_{3} f(x)=0, \quad \forall x \in R .
$$

Replacing $x$ by $x y$ in the first identity of (2.16) and using it again, we get $\lambda \epsilon_{1} c=$ $\epsilon_{1} f(x y)=\epsilon_{1}(f(x) y+x f(y))=\epsilon_{1} f(x) y+x \epsilon_{1} f(y)=\lambda \epsilon_{1} c y+x \lambda \epsilon_{1} c$; that is,

$$
\lambda \epsilon_{1} c=\lambda \epsilon_{1} c y+x \lambda \epsilon_{1} c \quad \forall x, y \in R .
$$

Replacing $y$ by $-x$ in (2.17), we get $\lambda \epsilon_{1} c=\lambda \epsilon_{1} c(-x)+x \lambda \epsilon_{1} c=-x \lambda \epsilon_{1} c+x \lambda \epsilon_{1} c=0$. Thus $\epsilon_{1} f(x)=\lambda \epsilon_{1} c=0$ for all $x \in R$. Hence, using (2.16), we get $f(x)=\left(\epsilon_{1}+\epsilon_{2}+\right.$ $\left.\epsilon_{3}\right) f(x)=\epsilon_{2} f(x)$, which implies $c f(x)=c \epsilon_{2} f(x)=\epsilon_{2} c f(x)=0$. Thus $c f(x)=0$ for all $x \in R$. Since $c g$ is central, therefore, analogously, it follows that $c g(x)=0$ for all $x \in R$. Hence $c f(x) x=0$ and $c x g(x)=0$ for all $x \in R$. Thus $c(f(x) x+x g(x))=0$. In particular, $0=c\left(f\left(x_{0}\right) x_{0}+x_{0} g\left(x_{0}\right)\right)=c^{2}$. Since a semiprime ring has no nonzero central nilpotents, therefore $c=0$; that is, $f\left(x_{0}\right) x_{0}+x_{0} g\left(x_{0}\right)=0$. Since $x_{0}$ is an arbitrary element of $R$, therefore

$$
f(x) x+x g(x)=0 \quad \forall x \in R .
$$

Using Theorem 1.1, from (2.18), we get that $f$ and $g$ are central.

Taking $g(x)=f(x)$ in Theorem 2.2 and considering (2.18), we get the following corollary.

COROLLARY 2.3. Let $f$ be a skew-centralizing derivation of a semiprime ring $R$, then $f$ is skew-commuting. 
COROLLARY 2.4. Let $R$ be a noncommutative prime ring and $f, g$ a pair of derivations of $R$ such that $f(x)+x g(x) \in Z(R)$ for all $x \in R$, then $f=g=0$.

Proof. Since $R$, being prime, is semiprime, therefore, by (2.18), we get $f(x) x+$ $x g(x)=0$ for all $x \in R$. Then Theorem 1.1 gives

$$
f(u)[x, y]=0=g(u)[x, y] \quad \forall u, x, y \in R
$$

Replacing $y$ by $z y$ in (2.19) and using (2.19) again, we get $f(u) z[x, y]=0=g(u) z[x$, $y$ ]. Since $R$ is prime and noncommutative, therefore $f(u)=0=g(u)$ for all $u \in R$. Thus $f=g=0$.

It is well known that there are no nonzero linear derivations on a commutative semisimple Banach algebra. Thus, it is natural to identify situations under which noncommutative semisimple Banach algebras do not admit nontrivial derivations. The following corollary, which follows as an application of our results, identifies such a situation.

COROLLARY 2.5. Let A be a noncommutative semisimple Banach algebra with center $Z(A)$ and let $f, g$ be a pair of linear derivations of $A$ such that $f(x) x+x g(x) \in Z(A)$ for all $x \in A$. Then $f=g=0$.

Proof. Since $A$ is semisimple, therefore it is semiprime. Thus, by Theorem 2.2, $f$ and $g$ are central and trivially commuting as well as centralizing. Hence, by [4, Corollary 3.7], $f$ and $g$ map $A$ into $Z(A) \cap \operatorname{rad}(A)$. Since $A$ is semisimple, therefore $\operatorname{rad}(A)=(0)$. Thus $f(x)=0=g(x)$ for all $x \in A$. Hence $f=0$ and $g=0$.

REMARK 2.6. (i) Taking $g(x)=f(x)$ in Corollary 2.5, we get that noncommutative semisimple Banach algebras do not admit nontrivial linear skew-centralizing derivations.

(ii) Taking $g(x)=f(x)$ in Theorem 2.2, we get that every skew-centralizing derivation $f$ of a semiprime ring $R$ is central.

ACKNOWLEDGMENTS. The authors gratefully acknowledge the support provided by King Fahd University of Petroleum and Minerals during this research. The authors would like to thank the referees for their valuable comments that led to the improvement of this note.

\section{REFERENCES}

[1] S. A. Amitsur, On rings of quotients, Symposia Mathematica, Vol. VIII (Convegno sulle Algebre Associative, INDAM, Rome, 1970), Academic Press, London, 1972, pp. 149-164.

[2] W. E. Baxter and W. S. Martindale III, Jordan homomorphisms of semiprime rings, J. Algebra 56 (1979), no. 2, 457-471.

[3] H. E. Bell and W. S. Martindale III, Centralizing mappings of semiprime rings, Canad. Math. Bull. 30 (1987), no. 1, 92-101.

[4] M. Brešar, Centralizing mappings on von Neumann algebras, Proc. Amer. Math. Soc. 111 (1991), no. 2, 501-510.

[5] _ Centralizing mappings and derivations in prime rings, J. Algebra 156 (1993), no. 2, 385-394. 
[6] _ On skew-commuting mappings of rings, Bull. Austral. Math. Soc. 47 (1993), no. 2, 291-296.

[7] _ On certain pairs of functions of semiprime rings, Proc. Amer. Math. Soc. 120 (1994), no. 3, 709-713.

[8] B. Hvala, Generalized derivations in rings, Comm. Algebra 26 (1998), no. 4, 1147-1166.

[9] A. B. Thaheem, On a pair of derivations on semiprime rings, to appear in Arch. Math. (Brno).

[10] A. B. Thaheem and M. S. Samman, A note on $\alpha$-derivations on semiprime rings, Demonstratio Math. 34 (2001), no. 4, 783-788.

Muhammad Anwar Chaudhry: Department of Mathematical Sciences, King Fahd University of Petroleum and Minerals, Dhahran 31261, Saudi Arabia

E-mail address: chaudhry@kfupm. edu.sa

A. B. Thaheem: Department of Mathematical Sciences, King Fahd University of Petroleum and Minerals, Dhahran 31261, Saudi Arabia

E-mail address: athaheem@kfupm.edu.sa 


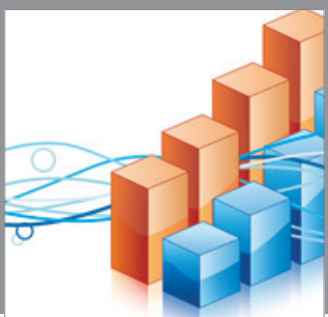

Advances in

Operations Research

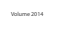

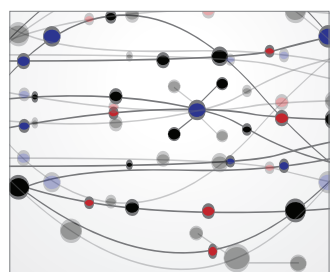

\section{The Scientific} World Journal
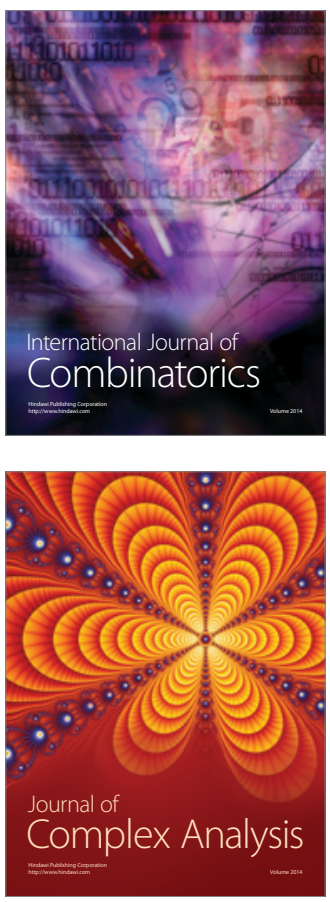

International Journal of

Mathematics and

Mathematical

Sciences
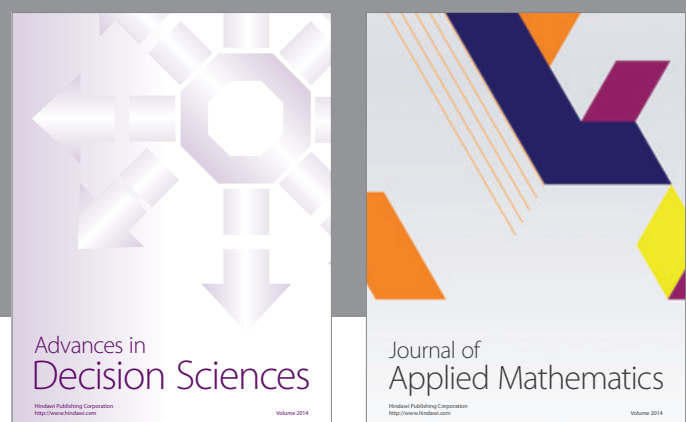

Journal of

Applied Mathematics
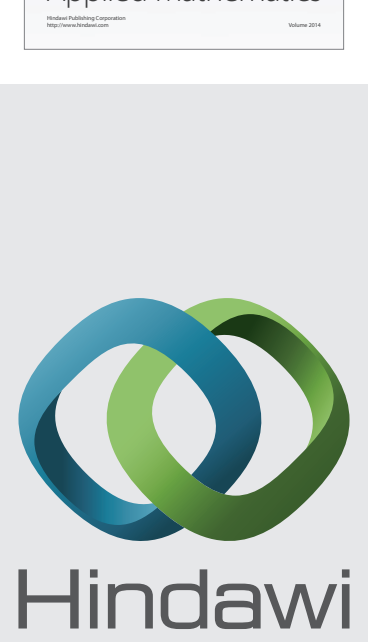

Submit your manuscripts at http://www.hindawi.com
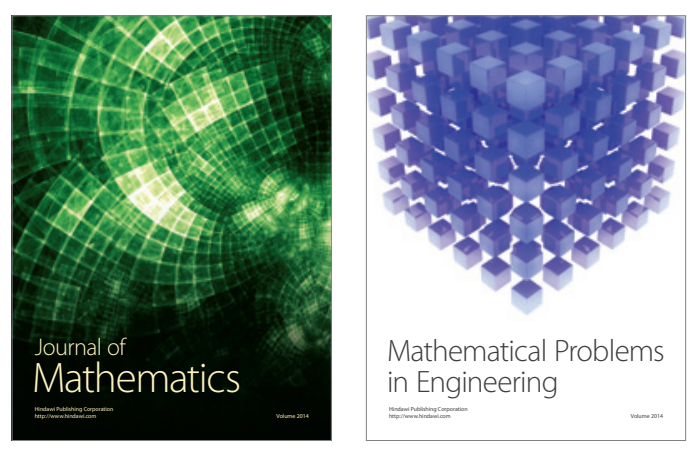

Mathematical Problems in Engineering
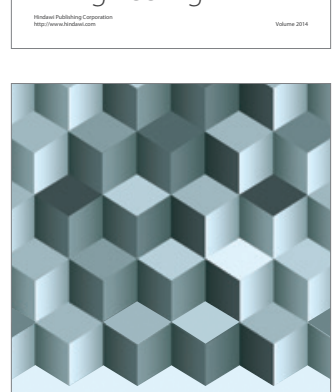

Journal of

Function Spaces
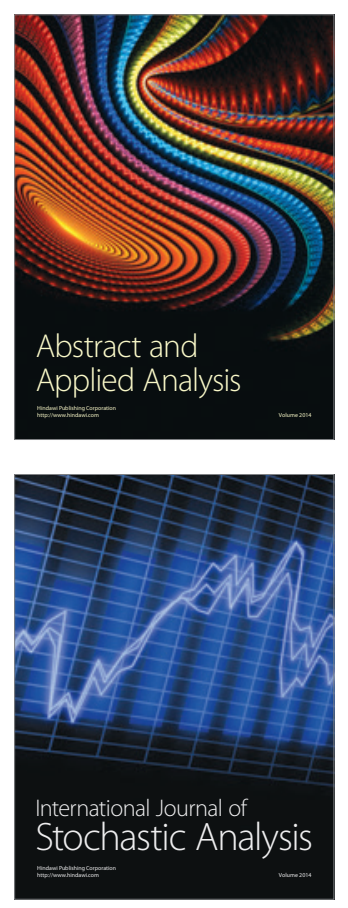

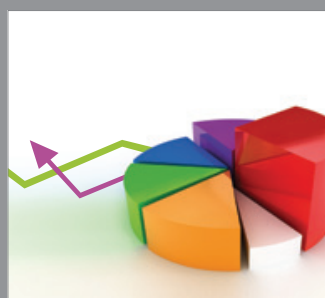

ournal of

Probability and Statistics

Promensencen
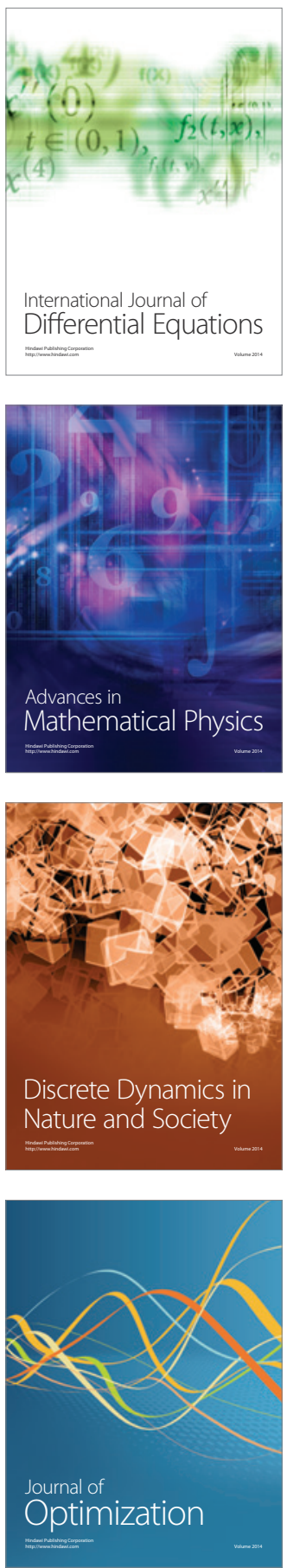